\title{
PSEUDOCOMPACT SPACES AND FUNCTIONALLY DETERMINED UNIFORMITIES
}

\author{
RODOLFO TALAMO
}

\begin{abstract}
A topological space is pseudocompact if and only if every admissible uniformity is functionally determined. We construct, on the discrete countable space $N$, an admissible (pseudo)-metric uniformity which is not functionally determined.
\end{abstract}

0. Introduction. It is well known that every precompact uniform space is also functionally determined (since a precompact space is weakly generated by all uniformly continuous real-valued mappings which are bounded). Moreover we know that a topological space is pseudocompact if and only if every admissible uniformity is precompact (see [4, 15Q]). Thus pseudocompactness implies that all admissible uniformities are functionally determined.

The following question now arises: Is the preceding implication invertible? In the present note we give a positive answer to this question, that is, we prove that a topological space is pseudocompact if and only if every admissible uniformity is functionally determined.

1. Basic concepts and results. We say that a uniform space is functionally determined if its uniformity is weakly generated by a collection of real-valued mappings. It may be easily seen that such spaces are representable as uniform subspaces of products of real lines, with the usual uniform structure.

Let $\varepsilon$ be a positive real number and $d$ a metric.

Definition. A finite set $F_{\varepsilon}=\left\{x_{0}, x_{1}, \ldots, x_{n}\right\}$ of points in $(X, d)$ is called an $\varepsilon$-chain of length $n$ if $d\left(x_{i-1}, x_{i}\right)<\varepsilon$ for all $i=1,2, \ldots, n$. A point $x \in X$ is $\varepsilon$-chained to a point $y \in X$ if there is an $\varepsilon$-chain $F_{\varepsilon}$ such that $x, y$ are in $F_{\varepsilon}$. A metric space $(X, d)$ is finitely chainable if, given $\varepsilon>0$, there is a finite set of points $P_{\varepsilon}=\left\{p_{1}, p_{2}, \ldots, p_{i}\right\}$ such that the length of the $\varepsilon$-chains to points of $P_{\varepsilon}$ is bounded. The above terminology is due to Atsuji [2].

We recall some useful results:

LEMMa 1.1 (sEe [2]). A metric space is finitely chainable iff it does not admit a uniformly continuous unbounded real mapping.

COROLlary 1.2. A finitely chainable nonprecompact metric space is not functionally determined.

Received by the editors June 30, 1975 and, in revised form, October 5, 1975.

AMS (MOS) subject classifications (1970). Primary 54C30, 54E15.

Key words and phrases. Pseudocompact space, functionally determined uniformity, $C$-embedding, $u$-embedding.

○ American Mathematical Society 1976 
We point out that the proof of this corollary utilizes the observation that a set of bounded real mappings weakly generates a precompact structure.

Proposition 1.3. If $(X, d)$ and $(Y, e)$ are finitely chainable (pseudo)-metric spaces, then $(X \times Y, d+e)$ is finitely chainable.

The verification is routine and we omit it.

Proposition 1.4. Let $X$ be a set, and $d$ and e finitely chainable metrics on $X$. Then the diagonal $\Delta$, in the relativization of the product metric $d+e$, is finitely chainable.

Proof. If $f$ is a uniformly continuous real-valued function on the diagonal $\Delta$, we extend $f$ to $X \times X$ by $\bar{f}(x, y)=f(x, x)$. Since $\bar{f}$ is bounded by Proposition 1.3 and Lemma 1.1 , so is $f$.

We finally recall (see [1]) that a subspace $X$ is said to be u-embedded in a topological space $S$ if every admissible uniformity on $X$ can be extended to an admissible uniformity on $S$. Every Lindelöf, closed, $C$-embedded subspace of a Tychonoff space is $u$-embedded (see [1, pp. 194-218]).

2. A characterization of pseudocompactness. The following is the main theorem.

THEOREM. A topological space is pseudocompact if and only if every admissible uniformity is functionally determined.

Proof. If $S$ is pseudocompact, then we know that every admissible uniformity is precompact. Since every precompact uniformity is functionally determined, the necessity of the condition is clear.

To prove its sufficiency, we consider the contrapositive and divide the proof into two steps.

(i) Construction of a nonfunctionally determined uniformity on the discrete countable space $N$. We consider first the space $\left(P, d_{1}\right)$ where $P$ is the set of all polynomial functions with rational coefficients which map $[0,1]$ into itself and $d_{1}$ is the usual metric of uniform convergence. The space $\left(P, d_{1}\right)$ is a countable, finitely chainable and not $d_{1}$-precompact metric space. (This may be shown by adapting the proof for $15 \mathrm{~L}$ of [4].)

Let us consider also the space $\left(P, d_{2}\right)$, where the underlying set is the same and $d_{2}$ is defined by

$$
d_{2}\left(f_{m}, f_{n}\right)=\|1 / m-1 / n\|,
$$

where $f_{m}, f_{n} \in P$, after some enumeration of $P$ by positive integers. This is a precompact metric which induces the discrete topology on $P$. We consider now the space $\left(P \times P, d_{1}+d_{2}\right)$ and its diagonal $\Delta$. For Propositions 1.3 and 1.4 the diagonal is finitely chainable in the relativization of the product metric. This space is not precompact because its metric uniformity say $d^{\prime}$, is finer than that generated by $d_{1}$ which is not precompact. Therefore, by Corollary 1.2, it is not functionally determined.

(ii) Every nonpseudocompact space admits a nonfunctionally determined uniformity.

Let $S$ be a nonpseudocompact topological space. It contains a $C$-embedded 
copy of $N$, the discrete countable space. Since $N$ is Lindelöf and since countable $C$-embedded subsets are closed, it follows that $N$ is also $u$ embedded. The extension to $S$ of the uniformity generated by $d^{\prime}$ on $N$ cannot be functionally determined, since every trace of a functionally determined uniformity is. This completes the proof.

Concluding remarks. An extension of the above can be obtained using the usual techniques of extending (pseudo)-metrics. For example, let $j$ be an isometric embedding of $\left(N, d^{\prime}\right)$ in the Banach space $C^{*}(N)$, and $j^{*}$ an extension of $j$ to $R$ (Tietze Generalized Extension Theorem). Then the metric on $R$ defined by

$$
r(x, y)=d^{*}\left(j^{*}(x), j^{*}(y)\right)
$$

where $d^{*}$ is the norm on $C^{*}(N)$, is a nonfunctionally determined metric on $R$. It would be interesting to obtain a characterization of uniformly continuous real mappings with respect to $r$ in "merely numerical" terms.

ACKNOWLEDGEMENT. The author wishes to thank the referee for his valuable comments.

\section{REFERENCES}

1. R. A. Alo and H. L. Shapiro, Normal topological spaces, Cambridge Univ. Press, Cambridge, 1974.

2. M. Atsuji, Uniform continuity of continuous functions of metric spaces, Pacific J. Math. 8 (1958), 11-16, 941. MR 20 \#5468.

3. J. Dugundji, Topology, Allyn and Bacon, Boston, Mass., 1968. MR 33 \# 1824.

4. L. Gillman and M. Jerison, Rings of continuous functions, University Ser. in Higher Math., Van Nostrand, Princeton, N. J., 1960. MR 22 \#6994.

Department of Mathematics, Carnegie-Mellon University, Pittsburgh, Pennsylvania 15213 (Current address)

Istituto di Geometria, Università di ToRino, Torino, Italia 